medgen $2017 \cdot 29: 234-238$

DOI 10.1007/s11825-017-0130-x

Online publiziert: 27. Juni 2017

(c) Der/die Autor(en) 2017. Dieser Artikel ist eine Open-Access-Publikation.

CrossMark

\author{
Britta Landfried ${ }^{1,4} \cdot$ Christian Grimm ${ }^{1,2,3,4}$ \\ ${ }^{1}$ Augenklinik, Universitätsspital Zürich, Zürich, Schweiz \\ ${ }^{2}$ Zentrum für integrative Humanphysiologie (ZIHP), Universität Zürich, Zürich, Schweiz \\ ${ }^{3}$ Zentrum für Neurowissenschaften Zürich (ZNZ), Universität Zürich, Zürich, Schweiz \\ ${ }^{4}$ Labor für Zellbiologie der Netzhaut, Augenklinik, Universität Zürich, Schlieren, Schweiz
}

\title{
Neuroprotektion geschädigter Photorezeptoren
}

\section{Präklinische Forschung}

\section{Neurotrophe Faktoren}

gemeinsame Endstrecke vieler Erkrankungen der Netzhaut, welche hierdurch letztendlich zu Erblindung führen. Dies gilt sowohl für erbliche Netzhautdystrophien als auch für multifaktorielle Krankheiten, wie die altersabhängige Makuladegeneration und andere Entitäten dieses Formenkreises. Obwohl es für die meisten dieser Krankheiten noch keine kurativen Therapieoptionen gibt, zeigen experimentelle Ansätze vielversprechende Resultate in präklinischen sowie zum Teil auch in klinischen Studien. Dazu gehören die Neuroprotektion, die Gentherapie, die Transplantation von Zellen, Sehprothesen [8] und die Optogenetik [35]. Die Neuroprotektion nimmt dabei eine Sonderstellung ein, da sie sowohl als Einzeltherapie wie auch in Kombination mit anderen Therapien einsetzbar ist. Die Entwicklung und Anwendung von effizienten, gut verträglichen und einfach zu verabreichenden neuroprotektiven Substanzen erscheint deshalb vorteilhaft, weil diese womöglich krankheitsübergreifend wirken und somit das Sehvermögen bei vielen Patienten erhalten oder dessen Verlust zumindest verzögert werden könnte. Eine Vielzahl relevanter Substanzen wurde in präklinischen Modellen identifiziert und untersucht. Einige wurden auch bereits in klinischen Versuchen, allerdings mit unterschiedlichem Erfolg, getestet.
Neurotrophe Faktoren können sowohl die Entwicklung und Differenzierung der neuronalen Zellen beeinflussen als auch deren Überleben in Stresssituationen fördern. $\mathrm{Zu}$ den wichtigsten neurotrophen Faktoren gehören die Mitglieder der FGF-(,fibroblast growth factor"), GDNF-(„glial cell line-derived neurotrophic factor"), IL-6-(interleukin-6), EGF-(„epidermal growth factor"), IGF(,insulin like growth factor"), TGFb("transforming growth factor beta") und Neurotrophin-Familien. Nicht alle Faktoren zeigen hierbei die gleiche Effizienz bei der Protektion der Sehzellen.

$\mathrm{Zu}$ den am besten charakterisierten Faktoren zählt CNTF (,ciliary neurotrophic factor"), ein zur IL-6-Familie gehörendes Zytokin. Die neuroprotektive Funktion von CNTF in der Netzhaut wurde bereits 1992 in der RCS(Royal College of Surgeon)-Ratte, einem etablierten Modell für die Netzhautdegeneration, beschrieben [25] und später in weiteren Tiermodellen bestätigt [40]. Interessanterweise scheint CNTF seine protektive Wirkung indirekt über Müller-Gliazellen zu entfalten. CNTF ist vor allem deshalb interessant, weil der Faktor neben Stäbchen auch Zapfen schützen kann und zudem die Regeneration der Zapfenaußensegmente fördert [27]. Vor diesem Hintergrund ist CNTF auch in Bezug auf den klinischen Einsatz attraktiv.

Gut beschrieben wurde auch das Schutzpotenzial von BDNF („,brain-derived neurotrophic factor") und GDNF.
Experimentelle Ansätze lassen vermuten, dass sowohl BDNF als auch GDNF ihre schützende Wirkung für Sehzellen wie CNTF über eine Aktivierung der Müller-Zellen entfalten könnten [17, 33]. Diese Daten rücken die MüllerGliazellen in den Fokus und etablieren sie als mögliche Zielzellen für die Aktivierung eines Programms zum Schutz der Sehzellen.

Neben CNTF war FGF2 oder bFGF einer der ersten neurotrophen Faktoren, für welchen eine protektive Wirkung für Sehzellen gezeigt wurde. Sowohl die subretinale als auch die intravitreale Injektion von bFGF in RCS Ratten bewirkte eine signifikante Verzögerung der degenerativen Prozesse in der Netzhaut und sicherte das Überleben der Sehzellen für mehrere Wochen [14]. In der Folge wurde die Schutzwirkung von bFGF auch in anderen Tiermodellen nachgewiesen [30]. Es konnte zudem gezeigt werden, dass bFGF auch endogen als zelluläre Antwort auf einen Sehzellschaden produziert wird und schützend wirkt [30].

"Pigment epithelium-derived factor" (PEDF) nimmt eine gewisse Sonderstellung unter den neuroprotektiven Faktoren ein, da PEDF sowohl Sehzellen schützen [19] als auch durch eine antiangiogene Wirkung der Blutgefäßbildung durch VEGF (,vascular endothelial growth factor") entgegenwirken kann [38]. Neben dem retinalen Pigmentepithel scheinen wiederum die Müller-Gliazellen den schützenden Faktor PEDF zu produzieren [12]. 


\section{Antioxidantien}

Oxidativer Stress ist eine permanente Gefahrenquelle für die Zellen der Netzhaut. Durch die hohe Sauerstoffkonzentration im Gewebe und die lebenslange Belastung durch Licht können freie Radikale entstehen, die Proteine und Lipide schädigen und dadurch die Funktion als auch die Lebensfähigkeit der Sehzellen beeinträchtigen können. Zudem führt die Phagozytose der abgestoßenen Sehzellenaußensegmente durch das retinale Pigmentepithel mit der Zeit zur Ansammlung von unverdaubarem oxidiertem Material, das zum Beispiel als Lipofuszin die Zellen schädigen kann. Durch diese ständige oxidative Belastung haben die Zellen eigene Schutzmaßnahmen entwickelt und produzieren Enzyme mit antioxidativer Wirkung. Daneben enthalten die RPE-Zellen auch Stoffe wie Melanin, Lutein und Zeaxanthin, welche überschüssiges Licht absorbieren und so vor der Bildung von Radikalen schützen [37].

Es ist daher nicht verwunderlich, dass exogen zugeführte antioxidative Substanzen einen Schutz für Sehzellen bieten können. Substanzen wie Resveratrol [23], Ginkgo-biloba-Extrakt [32], Berberin [34], $a$-Tocopherol, Ascorbinsäure, a-Liponsäure [22], N-Acetyl Cystein [26], Dimethylurea [32] und andere zeigen dabei eine protektive Wirkung im Modell der lichtinduzierten Sehzellapoptose und können zum Teil auch die Degeneration der Netzhaut in vererbten Modellen für Retinitis pigmentosa verzögern. Allerdings ist die Wirkungsweise vieler dieser Substanzen nicht eingehend untersucht worden und die Definition der Bandbreite des vermittelten Schutzes steht ebenfalls meistens noch aus.

Flavonoide bilden eine große Klasse von pflanzlichen Sekundärmetaboliten, die unter anderem vor UV-Strahlung schützen können. Viele von ihnen haben zudem eine antioxidative und/oder entzündungshemmende Wirkung. Zu den Flavonoiden mit protektiver Wirkung gehören Anthocyanide [31] und Flavanole wie Epigallocatechingallat [9].

Die Applikation von Nanopartikeln mit antioxidativer Wirkung könnte eine
Alternative zu den obigen Substanzen darstellen. Die Oxide von Cerium und Yttrium, Metallen der seltenen Erden, wurden erfolgreich als Antioxidantien bei Netzhautdegenerationen im Tiermodell eingesetzt [5, 28]. Insbesondere die Schutzwirkung von Ceriumoxid, besser bekannt unter dem Namen Nanoceria [5], wurde bereits in mehreren tierexperimentellen Ansätzen beschrieben.

\section{Neurosteroide}

Die Netzhaut synthetisiert neuroaktive Steroide, welche in verschiedene physiologische Prozesse eingreifen und auch in der Entwicklung eine wichtige Rolle spielen. Um diese Funktionen wahrnehmen zu können, binden die Steroide an spezifische Rezeptoren. So sind sowohl die „nuclear estrogen receptors“ (ERa und $\mathrm{ER} \beta$ ) wie auch Rezeptoren für Progesteron in der Netzhaut exprimiert $[4,20]$. Neben ihren physiologischen Funktionen wirken Steroide zudem protektiv. Besonders sind hierbei die schützenden Eigenschaften von 17-Estradiol (E2) und Progesteron hervorzuheben. Beide Steroide wurden im Tiermodell bereits mit Erfolg eingesetzt [20,43]. Zusätzlich wurde gezeigt, dass das synthetische Progesteron-Analog Norgestrel eine gute protektive Wirkung sowohl in induzierten als auch vererbten Modellen der Netzhautdegeneration zeigt [11]. Interessanterweise könnte diese Schutzwirkung endogene neurotrophe Faktoren wie „leukemia inhibitory factor" (LIF) miteinbeziehen [2], was darauf hindeutet, dass neuroprotektive Substanzen in der Netzhaut sowohl spezifische als auch gemeinsame Signalwege nutzen, um die neuronalen Zellen zu schützen.

\section{Rasagilin, Minocyclin und Rapamycin}

Rasagilin ist ein selektiver Monoaminoxidase (MAO) Hemmer, der vor allem in der Behandlung von Parkinson-Patienten zum Einsatz kommt. Nach oraler Verabreichung konnte Rasagilin caspaseabhängige Zelltodmechanismen in der Netzhaut unterdrücken und so die Degeneration der Sehzellen in der rds(„retinal degeneration slow“)-Maus verlangsamen
[13]. Minocyclin ist ein Antibiotikum, welches zu den Tetrazyklinen gehört und häufig zur Behandlung von Akne eingesetzt wird. Die systemische Behandlung von Mäusen mit Minocyclin konnte Sehzellen sowohl gegen Lichtschaden als auch gegen vererbte Degeneration schützen $[18,45]$. Rapamycin ist ein mTOR Inhibitor, der auch immunsuppressiv wirkt und in der Medizin unter anderem nach Organtransplantationen in einer Kombinationstherapie eingesetzt wird. In der Netzhaut konnte Rapamycin Sehzellen gegen Lichtschaden schützen. Der zugrunde liegende Mechanismus ist noch unklar, könnte aber eine differentielle Regulation der Autophagie beinhalten [24].

\section{Klinische Forschung}

Die Anzahl der klinischen Versuche mit neuroprotektiven Substanzen hat in den letzten Jahren signifikant zugenommen. Mögliche Applikationswege beinhalten die topische Gabe in Form von lokalen Augentropfen, die subkonjunktivale Applikation, die intravitreale Gabe sowie die orale Einnahme von Substanzen. Entsprechende Beispiele werden in den folgenden Absätzen mit den jeweiligen Substanzen thematisiert. Da aber die Resultate vieler dieser Studien noch nicht verfügbar sind, ist eine Beurteilung der Effektivität der Therapien häufig noch nicht möglich.

\section{CNTF}

Um erhöhte intraokulare Konzentrationen von CNTF über einen längeren Zeitraum zu ermöglichen, wurde ein Implantat entwickelt, welches immortalisierte und CNTF-produzierende Zellen mit einer semipermeablen Polymermembran umhüllt. Dieses Implantat (NT-501, Neurotech, Lincoln, RI) wird in den Glaskörper eingeführt und an der Sklera verankert. Die Zellen werden über den Glaskörper mit Nährstoffen und Sauerstoff versorgt und überleben daher über einen längeren Zeitraum. Währenddessen produzieren sie kontinuierlich humanes CNTF, ohne dass bislang schwerwiegende Nebenwirkungen festgestellt wurden [21]. 
NT-501 wurde bereits bei Retinitis pigmentosa im Früh- und Spätstadium untersucht. Die erhöhten CNTF-Mengen führten zwar zu einer Zunahme der retinalen Schichtdicke, ohne dass jedoch ein Anstieg des Visus festgestellt werden konnte. In der Hochdosisgruppe wurde sogar ein Abfall der Netzhautsensitivität festgestellt, welcher aber nach Explantation des Implantates komplett reversibel war [1]. Gleichfalls negative Resultate wurden für NT-501 in einer Phase I/IIStudie bei CNBG3 Achromatopsie Patienten erhalten (NCT01648452) [44]. Die Ergebnisse einer neu initiierten Phase IIStudie (NCT01530659), welche den Effekt von NT-501 bei früher Retinitis pigmentosa und Usher-Syndrom Typ 2 und 3 untersucht, bleiben abzuwarten.

Positive Ergebnisse mit NT-501 wurden hingegen bei Patienten mit der trockenen Form der altersabhängigen Makuladegeneration oder geografischer Atrophie erzielt. In einer Phase II-Studie konnte gezeigt werden, dass eine hohe Dosis von CNTF bei diesen Patienten sowohl die Netzhautdicke verstärken als auch die Sehschärfe über einen Zeitraum von zwölf Monaten stabilisieren konnte [46]. Obwohl dies auf eine mögliche Anwendung von NT-501 bei der trockenen AMD hindeuten könnte, wurden keine weiteren Daten hierzu veröffentlicht. Die Firma scheint sich mit ihrem Produkt neu auf makuläre Telangiektasie (MacTel) und Glaukom zu fokussieren (http://www.neurotechusa. com/4-29-16_neurotech_announces_ renewedfocusNT501CNTProgram.pdf).

\section{PEDF}

Um die Mengen von PEDF im humanen Auge zu erhöhen, wurde ein Adenovirus, welcher PEDF exprimiert (AdPEDF.11), Patienten mit exudativer AMD intravitreal appliziert. Dieses Vorgehen zeigte bereits im Tiermodell gute Ergebnisse $[19,29]$ und hat auch bei den Patienten $\mathrm{zu}$ einer antiangiogenen Wirkung geführt. Eine Progredienz der Läsionen war in derjenigen Patientengruppe mit hochdosierter PEDF-Gabe über einen Zeitraum von zwölf Monaten nicht mehr festzustellen [3]. Drei neue klinische Versuche (NCT03022318; NCT03023059;
NCT02873351) verfolgen einen interessanten alternativen Ansatz, um einen PEDF-vermittelten Schutz der Sehzellen $\mathrm{zu}$ erzielen. Dabei soll Patienten mit mittlerer bis fortgeschrittener AMD L-DOPA in Tablettenform verabreicht werden, um durch die Aktivierung von GPR143 im RPE das Verhältnis von VEGF zu PEDF zugunsten von PEDF $\mathrm{zu}$ verändern.

\section{Antioxidantien}

Eine große Studie $\mathrm{zu}$ altersbedingten Augenerkrankungen (AREDS) testete die orale Einnahme einer hochdosierten Mixtur aus Antioxidantien (Vitamine A, C und E), Zink und Kupfer. Das Ergebnis zeigte, dass dadurch das Risiko innerhalb von fünf Jahren aus einer frühen eine fortgeschrittene Form der AMD zu entwickeln um $25 \%$ verringert wurde [6]. Eine zusätzliche Gabe von Lutein, Zeaxanthin und Omega-3-Fettsäuren führte zu keiner weiteren Verbesserung [7].

OT-551, ein lipophiles Hydroxylaminpräparat, welches $\mathrm{zu}$ seinem aktiven Metaboliten TEMPOL-H verstoffwechselt wird, kann direkt oder indirekt mit freien Radikalen reagieren und zeigte antioxidative Effekte und einen Schutz der Photorezeptoren im Tiermodell [36]. In einer Phase II-Studie wurde OT-551 topisch dreimal täglich für insgesamt zwei Jahre an einem Auge verabreicht. Es zeigte sich, dass acht von zehn Studienteilnehmern im Studienauge weniger Buchstaben auf der EDTRS-Scala verloren als im Kontrollauge. Allerdings konnte kein Einfluss auf die Progression der geografischen Atrophie oder auf die Kontrastsensitivität gezeigt werden, sodass sich ein neuroprotektiver Effekt von OT-551 in humanen Augen insgesamt nicht bestätigen ließ [42].

\section{Rasagilin, Minocyclin und Rapamycin}

Der MAO-Hemmer Rasagilin und das Antibiotikum Minocyclin werden momentan in einer prospektiven Studie zum Schutz der Sehzellen bei Netzhautablösung getestet (NCT02068625, NCT01297816). Die Ergebnisse beider medgen $2017 \cdot 29: 234-238$

DOI 10.1007/s11825-017-0130-x

(c) Der/die Autor(en) 2017. Dieser Artikel ist eine Open-Access-Publikation.

\section{B. Landfried · C. Grimm}

\section{Neuroprotektion geschädigter Photorezeptoren}

\section{Zusammenfassung}

Der Schutz der Sehzellen durch Neuroprotektion ist ein vielversprechender Ansatz, der bei vielen degenerativen Netzhauterkrankungen entweder als Monooder Kombinationstherapie zum Einsatz kommen könnte. Viele neuroprotektive Substanzen wurden im Tiermodell identifiziert und erfolgreich getestet. Einige dieser Substanzen wurden auch bereits in klinischen Versuchen am Patienten untersucht, allerdings mit unterschiedlichem Erfolg. Diverse Versuchsansätze werden derzeit überprüft.

\section{Schlüsselwörter}

Neuroprotektion · Netzhaut · Sehzellen . Präklinische Modelle · Klinische Versuche

\section{Neuroprotection of damaged photoreceptors}

\section{Abstract}

Neuroprotection of photoreceptor cells is a promising approach that could be used to treat degenerative retinal diseases, either as mono- or combination therapy. Many neuroprotective substances have been identified and successfully tested in animal models. Several of them have already been investigated in clinical trials involving patients as well, although with variable success. Various experimental approaches are currently being reviewed.

\section{Keywords}

Neuroprotection - Retina · Photoreceptors . Pre-clinical models · Clinical trials

Studien sind aktuell noch ausstehend. Minocyclin wurde aber bereits in Bezug auf die Reduktion eines diabetischen Makulaödems untersucht. Eine kleinere Phase I/II-Studie zeigte einen Anstieg des Visus um durchschnittlich 5,8 \pm 5,4 Buchstaben bei einer gleichzeitigen Reduktion der Netzhautdicke um 8,1\% [10]. Da die Studie nur fünf Patienten und keine Placebogruppe umfasste, müssen diese Ergebnisse noch bestätigt werden. Die Wirksamkeit und Sicher- 
heit von Minocyclin wird derzeit noch in drei anderen klinischen Phase II-Studien bei geografischer Atrophie, fortgeschrittener Makuladegeneration sowie bei retinalen Zentralvenen- und Venenastverschlüssen evaluiert (NCT02564978, NCT01468844, NCT01468831). Wichtig scheint hier vor allem der Sicherheitsaspekt zu sein, da für Minocyclin eine gewisse Netzhauttoxizität in Maus [16] und Kaninchen [15] nachgewiesen wurde.

Da Autophagiemarker in humanen Drusen und gestressten RPE-Zellen gefunden wurden [39], wurde der mTORInhibitor Rapamycin in einer Phase I/IIStudie in Bezug auf die Progression der geografischen Atrophie untersucht. Eine subkonjuntivale Injektion von synthetischem Rapamycin (Sirolimus) alle drei Monate hatte keine feststellbaren positiven Effekte. Weder Sehschärfe, Netzhautdicke oder die Größe der Drusen waren signifikant unterschiedlich $\mathrm{zu}$ den Kontrollaugen [41].

\section{Fazit für die Praxis}

Obwohl neuroprotektive Ansätze in Tiermodellen gute Ergebnisse erzielten und somit verheißungsvoll für eine klinische Anwendung erscheinen, gestaltet sich die klinische Umsetzung für den Einsatz bei Patienten noch relativ schwierig. Einerseits stellt die notwendige langfristige und kontinuierliche Applikation der pharmazeutischen Substanzen eine große Herausforderung dar. Andererseits sind die humanen Pathomechanismen bei Netzhautdegenerationen immer noch nicht genügend untersucht, um sie in eine direkte Korrelation zu den Erkenntnissen aus den tierexperimentellen Ansätzen setzen zu können. Außerdem ist die Bestimmung der klinischen Endpunkte bei langsam verlaufenden Netzhautdegenerationen schwierig und kann eine jahrelange Untersuchungsspanne beanspruchen. Eine Identifikation von Parametern, die schon früh einen protektiven Effekt erkennen lassen, wäre daher von großem Interesse und könnte das Gebiet signifikant voranbringen.

\section{Korrespondenzadresse}

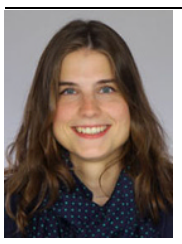

Dr. B. Landfried, MD

Labor für Zellbiologie der Netzhaut, Augenklinik, Universität Zürich Wagistrasse 14 8952 Schlieren, Schweiz Britta.Landfried@usz.ch

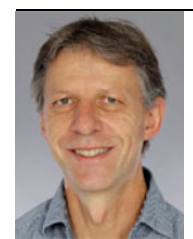

Prof. Dr. C. Grimm, PhD Labor für Zellbiologie der Netzhaut, Augenklinik, Universität Zürich Wagistrasse 14, 8952 Schlieren, Schweiz cgrimm@opht.uzh.ch

\section{Einhaltung ethischer Richtlinien}

Interessenkonflikt. B. Landfried und C. Grimm geben an, dass kein Interessenkonflikt besteht.

Dieser Beitrag beinhaltet keine von den Autoren durchgeführten Studien an Menschen oder Tieren.

Open Access. Dieser Artikel wird unter der Creative Commons Namensnennung 4.0 International Lizenz (http://creativecommons.org/licenses/by/4.0/deed. de) veröffentlicht, welche die Nutzung, Vervielfältigung, Bearbeitung, Verbreitung und Wiedergabe in jeglichem Medium und Format erlaubt, sofern Sie den/die ursprünglichen Autor(en) und die Quelle ordnungsgemäßnennen, einen Linkzur Creative Commons Lizenz beifügen und angeben, ob Änderungen vorgenommen wurden.

\section{Literatur}

1. Birch DG, Weleber RG, Duncan JL, Jaffe GJ, Tao W, Ciliary Neurotrophic Factor Retinitis Pigmentosa Study Groups (2013) Randomized trial of ciliary neurotrophic factor delivered by encapsulated cell intraocular implants for retinitis pigmentosa. Am J Ophthalmol 156(2):283-292.e1

2. Byrne AM, Roche SL, Ruiz-Lopez AM, Jackson AC, CotterTG (2016) The synthetic progestin norgestre acts to increase LIF levels in the rd10 mouse model of retinitis pigmentosa. Mol Vis 22:264-274

3. Campochiaro PA, Nguyen QD, Shah SM, Klein ML, Holz E, Frank RN, Saperstein DA, Gupta A, Stout JT, Macko J, DiBartolomeo R, Wei LL (2006) Adenoviral vector-delivered pigment epitheliumderived factor for neovascular age-related macular degeneration: results of a phase I clinical trial. Hum Gene Ther 17(2):167-176

4. Cascio C, Deidda I, Russo D, Guarneri P (2015) The estrogenic retina: The potential contribution to healthy aging and age-related neurodegenerative diseases of the retina. Steroids 103:31-41

5. Chen J, Patil S, Seal S, McGinnis JF (2006) Rare earth nanoparticles prevent retinal degeneration induced by intracellular peroxides. Nat Nanotechnol 1(2):142-150

6. Chew EY, Clemons TE, Agrón E, Sperduto RD, Sangiovanni JP, Kurinij N, Davis MD, Age-Related Eye Disease Study Research Group (2013) Long-term effects of vitamins $C$ and $E, \beta$-carotene, and zinc on age-related macular degeneration: AREDS report no. 35. Ophthalmology 120(8):1604-1611.e4

7. Chew EY, Clemons TE, Sangiovanni JP, Danis RP, Ferris FL, Elman MJ, Antoszyk AN, Ruby AJ, Orth D, Bressler SB, Fish GE, Hubbard GB, Klein ML, Chandra SR, Blodi BA, Domalpally A, Friberg T, Wong WT, Rosenfeld PJ, Agrón E, Toth CA, Bernstein PS, Sperduto RD (2014) Secondary analyses of the effects of lutein/zeaxanthin on age-related macular degeneration progression: AREDS2 report No.3. JAMA Ophthalmol 132(2):142-149

8. Chuang AT, Margo CE, Greenberg PB (2014) Retinal implants: a systematic review. Br J Ophthalmol 98(7):852-856

9. Costa BL, Fawcett R, Li GY, Safa R, Osborne NN (2008) Orally administered epigallocatechin gallate attenuates light-induced photoreceptor damage. Brain Res Bull 76(4):412-423

10. Cukras CA, Petrou P, Chew EY, Meyerle CB, Wong WT (2012) Oral minocycline for the treatment of diabetic macular edema (DME): results of a phase I/II clinical study. Invest Ophthalmol Vis Sci 53(7):3865-3874

11. Doonan F, O'Driscoll C, Kenna P, Cotter TG (2011) Enhancing survival of photoreceptor cells in vivo using the synthetic progestin Norgestrel. JNeurochem 118(5):915-927

12. Eichler W, Yafai $Y$, Keller T, Wiedemann $P$, Reichenbach A (2004) PEDF derived from glial Müller cells: a possible regulator of retinal angiogenesis. Exp Cell Res 299(1):68-78

13. Eigeldinger-Berthou S, Meier C, Zulliger R, Lecaudé S, Enzmann V, Sarra GM (2012) Rasagiline interferes with neurodegeneration in the Prph2/rds mouse. Retina (Philadelphia, Pa) 32(3):617-628

14. FaktorovichEG, Steinberg RH, Yasumura D, Matthes MT, LaVail MM (1990) Photoreceptor degeneration in inherited retinal dystrophy delayed by basic fibroblast growth factor. Nature 347(6288):83-86

15. Falavarjani KG, Pourhabibi A, Aghdam KA, Hosseini SB, Modarres M, Pazouki A, Khanamiri HN (2016) Determination of the toxicity of intravitreal minocycline in rabbit eyes. Cutan Ocul Toxicol 35(3):233-236

16. Ferrer-Martín RM, Martín-Oliva D, Sierra-Martín A, Carrasco MC, Martín-Estebané M, Calvente R, Martín-Guerrero SM, Marín-Teva JL, Navascués J, Cuadros MA (2015) Microglial activation promotes cell survival in Organotypic cultures of postnatal mouse retinal explants. PLoSOne 10(8):e0135238

17. Harada C, Harada T, Quah HM, Maekawa F, Yoshida K, Ohno S, Wada K, Parada LF, Tanaka K (2003) Potential role of glial cell line-derived neurotrophic factor receptors in Müller glial cells during light-induced retinal degeneration. Neuroscience 122(1):229-235

18. Hughes EH, Schlichtenbrede FC, Murphy CC, Broderick C, van Rooijen N, Ali RR, Dick AD (2004) Minocycline delays photoreceptor death in the rds mouse through a microglia-independent mechanism. ExpEye Res 78(6):1077-1084

19. Imai D, Yoneya S, Gehlbach PL, WeiLL, MoriK(2005) Intraocular gene transfer of pigment epitheliumderived factor rescues photoreceptors from lightinduced cell death. J Cell Physiol 202(2):570-578

20. Jackson AC, Roche SL, Byrne AM, Ruiz-Lopez AM, Cotter TG (2016) Progesterone receptor signalling in retinal photoreceptor neuroprotection. JNeurochem 136(1):63-77

21. Kauper K, McGovern $C$, Sherman $S$, Heatherton P, Rapoza R, Stabila P, Dean B, Lee A, Borges S, Bouchard B, Tao W (2012) Two-year intraocular delivery of ciliary neurotrophic factor by en- 
capsulated cell technology implants in patients with chronic retinal degenerative diseases. Invest Ophthalmol Vis Sci 53(12):7484-7491

22. Komeima K, Rogers BS, Campochiaro PA (2007) Antioxidants slow photoreceptor cell death in mouse models of retinitis pigmentosa. J Cell Physiol 213(3):809-815

23. Kubota S, Kurihara T, Ebinuma M, Kubota M, Yuki K, Sasaki M, Noda K, Ozawa Y, Oike Y, Ishida S, Tsubota K (2010) Resveratrol prevents light-induced retina degeneration via suppressing activator protein-1 activation. Am J Pathol 177(4):1725-1731

24. Kunchithapautham K, Coughlin B, Lemasters JJ, Rohrer B (2011) Differential effects of rapamycin on rods and cones during light-induced stress in albino mice. Invest Ophthalmol Vis Sci 52(6):2967-2975

25. LaVail MM, Unoki K, Yasumura D, Matthes MT, Yancopoulos GD, Steinberg RH (1992) Multiple growth factors, cytokines, and neurotrophins rescue photoreceptors from the damaging effects of constant light. Proc Natl Acad Sci U S A 89(23):11249-11253

26. Lee SY, Usui S, Zafar AB, Oveson BC, Jo YJ, Lu L, Masoudi S, Campochiaro PA (2011) N-Acetylcysteine promotes long-term survival of cones in a model of retinitis pigmentosa. J Cell Physiol 226(7):1843-1849

27. Li Y, Tao W, Luo L, Huang D, Kauper K, Stabila P, Lavail MM, Laties AM, Wen R (2010) CNTF induces regeneration of cone outer segments in a rat model of retinal degeneration. PLoSOne 5(3):e9495

28. Mitra RN, Merwin MJ, Han Z, Conley SM, Al-Ubaidi MR, Naash MI (2014) Yttrium oxide nanoparticles prevent photoreceptor death in a light-damage model of retinal degeneration. Free Radic Biol Med 75:140-148

29. Mori K, Gehlbach P, Ando A, McVey D, Wei L, Campochiaro PA (2002) Regression of ocular neovascularization in response to increased expression of pigment epithelium-derived factor. Invest Ophthalmol Vis Sci 43(7):2428-2434

30. O'Driscoll C, O'Connor J, O'Brien CJ, Cotter TG (2008) Basic fibroblast growth factor-induced protection from light damage in the mouse retina in vivo. J Neurochem 105(2):524-536

31. Paik SS, Jeong E, Jung SW, Ha TJ, Kang S, Sim S, Jeon JH, Chun MH, Kim IB (2012) Anthocyanins from the seed coat of black soybean reduce retinal degeneration induced by $\mathrm{N}$-methyl- $\mathrm{N}$ nitrosourea. Exp Eye Res 97(1):55-62

32. Ranchon I, Gorrand JM, Cluzel J, Droy-Lefaix MT, Doly M (1999) Functional protection of photoreceptors from light-induced damage by dimethylthiourea and Ginkgo biloba extract. Invest Ophthalmol Vis Sci 40(6):1191-1199

33. Saito T, Abe T, Wakusawa R, Sato $H$, Asai $H$, TokitaIshikawa Y, Nishida K (2009) TrkB-T1 receptors on Muller cells play critical role in brain-derived neurotrophic factor-mediated photoreceptor protection against phototoxicity. Curr Eye Res 34(7):580-588

34. Song D, Song J, Wang C, Li Y, Dunaief JL (2016) Berberine protects against light-induced photoreceptor degeneration in the mouse retina. ExpEye Res 145:1-9

35. Swiersy A, Klapper SD, Busskamp V (2017) Optogenetics: a therapeutic option for advanced retinal dystrophies. Klin Monbl Augenheilkd 234(3):335-342

36. Tanito M, Li F, Anderson RE (2010) Protection of retinal pigment epithelium by OT-551 and its metabolite TEMPOL-H against light-induced damage in rats. Exp Eye Res 91(1):111-114
37. Tokarz P, Kaarniranta K, Blasiak J (2013) Role of antioxidant enzymes and small molecular weight antioxidants in the pathogenesis of age-related macular degeneration (AMD). Biogerontology 14(5):461-482

38. Tombran-Tink J (2010) PEDF in angiogenic eye diseases. Curr Mol Med 10(3):267-278

39. Wang AL, Lukas TJ, Yuan M, Du N, Tso MO, Neufeld $\mathrm{AH}$ (2009) Autophagy and exosomes in the aged retinal pigment epithelium: possible relevance to drusen formation and age-related macular degeneration. PLoSOne 4(1):e4160

40. Wen R, Tao W, Li Y, Sieving PA (2012) CNTF and retina. Prog Retin Eye Res 31(2):136-151

41. Wong WT, Dresner S, Forooghian F, Glaser T, Doss L, Zhou M, Cunningham D, Shimel K, Harrington M, Hammel K, Cukras CA, Ferris FL, Chew EY (2013) Treatment of geographic atrophy with subconjunctival sirolimus: results of a phase I/II clinical trial. Invest Ophthalmol Vis Sc 54(4):2941-2950

42. Wong WT, Kam W, Cunningham D, Harrington M, Hammel K, Meyerle CB, Cukras C, Chew EY, Sadda SR, Ferris FL (2010) Treatment of geographic atrophy by the topical administration of OT-551: results of a phase II clinical trial. Invest Ophthalmol Vis Sci 51(12):6131-6139

43. Yu X, Rajala RV, McGinnis JF, Li F, Anderson RE, Yan X, Li S, Elias RV, Knapp RR, Zhou X, Cao W (2004) Involvement of insulin/phosphoinositide 3-kinase/Akt signal pathway in 17 beta-estradiol-mediated neuroprotection. J Biol Chem 279(13):13086-13094

44. Zein WM, Jeffrey BG, Wiley HE, Turriff AE, Tumminia SJ, Tao W, Bush RA, Marangoni D, Wen R, Wei LL, Sieving PA (2014) CNGB3-achromatopsia clinical trial with CNTF: diminished rod pathway responses with no evidence of improvement in conefunction. Invest Ophthalmol Vis Sci 55(10):6301-6308

45. Zhang C, Lei B, Lam TT, Yang F, Sinha D, Tso MO (2004) Neuroprotection of photoreceptors by minocycline in light-induced retinal degeneration. Invest Ophthalmol Vis Sci 45(8):2753-2759

46. Zhang K, Hopkins JJ, Heier JS, Birch DG, Halperin LS, Albini TA, Brown DM, Jaffe GJ, Tao W, Williams GA (2011) Ciliary neurotrophic factor delivered by encapsulated cell intraocular implants for treatment of geographic atrophy in age-related macular degeneration. Proc Natl Acad Sci U S A 108(15):6241-6245 